Draft version August 27, 2018

Preprint typeset using $\mathrm{L}^{\mathrm{A}} \mathrm{T} \mathrm{E}$. style emulateapj v. 04/17/13

\title{
EXPECTED NUMBER OF MASSIVE GALAXY RELICS IN THE PRESENT-DAY UNIVERSE
}

\author{
VICENT QUILIS \\ Departament d'Astronomia i Astrofísica, Universitat de València, 46100 - Burjassot, València, Spain \\ AND \\ IGNACiO TRUJiLlo ${ }^{1}$ \\ Instituto de Astrofísica de Canarias, c/ Vía Láctea s/n, E38205 - La Laguna, Tenerife, Spain \\ Draft version August 27, 2018
}

\begin{abstract}
The number of present-day massive galaxies that has survived untouched since their formation at high-z is an important observational constraint to the hierarchical galaxy formation models. Using three different semianalytical models based on the Millenium simulation, we quantify the expected fraction and number densities of the massive galaxies form at $z>2$ which have evolved in stellar mass less than $10 \%$ and $30 \%$. We find that only a small fraction of the massive galaxies already form at $\mathrm{z} \sim 2$ have remained almost unaltered since their formation $\left(<2 \%\right.$ with $\Delta \mathrm{M}_{\star} / \mathrm{M}_{\star}<0.1$ and $<8 \%$ with $\left.\Delta \mathrm{M}_{\star} / \mathrm{M}_{\star}<0.3\right)$. These fractions correspond to the following number densities of massive relics in the present-day Universe: $\sim 1.2 \times 10^{-6} \mathrm{Mpc}^{-3}$ for $\Delta \mathrm{M}_{\star} / \mathrm{M}_{\star}<0.1$ and $\sim 5.7 \times 10^{-6} \mathrm{Mpc}^{-3}$ for $\Delta \mathrm{M}_{\star} / \mathrm{M}_{\star}<0.3$. The observed number of relic candidates found in the nearby Universe is today pretty uncertain (with uncertainties up to a factor of $\sim 100$ ) preventing to establish a firm conclusion about the goodness of current theoretical expectations to predict such important number.

Subject headings: dark matter — galaxies: halos — galaxies: formation — galaxies: evolution
\end{abstract}

\section{INTRODUCTION}

Merging of galaxies is an intrinsically stochastic process. That means that there should be a number of galaxies formed in the early epochs of the Universe that has remained untouched until today. Identifying and exploring these relics objects is of fundamental relevance to understand the conditions and properties of the primordial phases of galaxy formation. But, how many of these objects are theoretically expected to survive without being significantly modified since high redshift? In this letter, we address this issue focusing our attention to the most massive $\left(\mathrm{M}_{\star}>8 \times 10^{10} \mathrm{M}_{\odot}\right)$ galaxies in the Universe.

Observationally, massive galaxies have been found to be more compact at high redshift (Daddi et al. 2005; Trujillo et al. 2006). Particularly those with spheroidlike morphologies show sizes (measured using their effective radii) a factor of $\sim 4$ more smaller at $\mathrm{z} \sim 2$ (see e.g. Trujillo et al. 2007; Buitrago et al. 2008). These massive compact galaxies are expected to grow as cosmic time increases both in stellar mass and in size by a continuous accretion of minor satellites (e.g. Bezanson et al. 2009; Hopkins et al. 2009). This channel of evolution agrees very well with many observations and it has been proven theoretically able to produce the expected size evolution (e.g. Naab et al. 2009; Sommer-Larsen \& Toft 2010, Feldmann et al. 2010, Oser et al. 2012). Being the merging a stochastic process, it is expected that a number of these massive compact galaxies remain unaltered since their formation. Consequently, quantifying the number of these massive old compact objects in the present-day Universe is a proxy to explore the number of massive relics of the early Universe.

Contradictory claims in relation to the number of to-

vicent.quilis@uv.es

${ }^{1}$ Departamento de Astrofísica, Universidad de La Laguna, E38205 La Laguna, Tenerife, Spain day massive compact galaxies with old stellar populations have been reported. On one hand, Trujillo et al. (2009) using the Sloan Digital Sky Survey (SDSS) did not find any clear evidence for a single massive "old" galaxy which has survived untouched since their high-z formation. In fact, they found that the number of massive $\left(\mathrm{M}_{\star}>8 \times 10^{10} \mathrm{M}_{\odot}\right)$ and compact $\left(\mathrm{r}_{e}<1.5 \mathrm{kpc}\right)$ galaxies in the nearby Universe $(\mathrm{z}<0.2)$ is less than $0.03 \%$ (see also Taylor et al. 2010 for a confirmation of this result). Moreover, these galaxies are not old but relatively young ( $\sim 2$ Gyr; see also Ferré-Mateu et al. 2012). On the other hand, Poggianti et al. (2013) using a much more modest area find evidence for up to 4 old massive compact galaxies satisfying the above selection criteria of stellar mass and size. The reason behind these two conflicting results remains a mystery and further studies are necessary to clarify this point.

From the theoretical perspective, there are not single quantification about the expected number of massive galaxies which should not have suffered any grow since their formation. Answering quantitatively this question is crucial if we want to settle the minor merging scenario (the present-day favoured channel of growth) under solid grounds. Intuitively, a moderate low number of massive compact galaxies in the nearby Universe should favour the hypothesis that massive galaxies have evolved by satellite accretion. However, if the number of today relics is very small or nil this will put into question the minor merging hypothesis. This is again due to the intrinsic stochastic nature of the merging model. It is then an urgent question to quantify which is the exact theoretical prediction about the expected number of massive relics in the present-day universe. Fortunately, current cosmological simulations are large enough to permit the estimation of this fraction with accuracy.

In this Letter, we explore the model predictions on the 
fraction and number density of massive galaxies form at $\mathrm{z}>2$ which have not increased their stellar mass since that epoch by more than $10 \%$ and $30 \%$. To quantify these numbers we have used three different semi-analytical models (DeLucia et al. 2007, Guo et al. 2011 and Guo et al. 2013) based on the Millennium simulation.

\section{GALAXY CATALOGUES}

We use the public release of a two very large N-body simulations, Millennium I (Springel et al. 2005) (MI) and Millennium I-WMAP7 (Guo et al. 2013) (MI7), a version of the original Millenium I simulation run using the seven-year WMAP data (Komatsu et al. 2011). The cosmological parameters for MI (MI7) simulation are: $\Omega_{m}=0.25\left(\Omega_{m}=0.272\right), \Omega_{b}=0.045\left(\Omega_{b}=\right.$ $0.045), \Omega_{\Lambda}=0.75\left(\Omega_{\Lambda}=0.728\right), n=1(n=0.961)$, $\sigma_{8}=0.9\left(\sigma_{8}=0.807\right), H_{0}=73 \mathrm{kms}^{-1} \mathrm{Mpc}^{-1}\left(H_{0}=\right.$ $\left.70.4 \mathrm{kms}^{-1} \mathrm{Mpc}^{-1}\right)$. The two simulations use the same number of particles, $2160^{3}$. Thus, the computational box has sides of $685 \mathrm{Mpc}(710 \mathrm{Mpc})$, and particles masses of $1.18 \times 10^{9} M_{\odot}\left(1.32 \times 10^{9} M_{\odot}\right)$. In order to facilitate the comparison between the three different catalogues and the observational data (see Sec. 4), we present all the results assuming $H_{0}=70 \mathrm{kms}^{-1} \mathrm{Mpc}^{-1}$.

A combination of two halo finders, a friends-offriends (FoF) by Davis et al. (1985) and SUBFIND (Springel et al. 2001), is used in order to analyze the simulations and to build up the dark matter merger trees. The dark matter haloes found in the N-body simulations are transformed into galaxies according to different semi-analytical models. Depending on the particular implementation of each model, several phenomenological recipes are used to produce the gas and stellar components in the virtual galaxies. In this Letter, we use three semi-analytical models available in the Millennium database web (Lemson et al. 2006).

The first one is the model by De Lucia \& Blaizot (2007) and the other two, in fact they are the same model but applied to different simulations, are Guo et al. (2011) and Guo et al. (2013). All of them are very similar being the models by Guo et al. (2011) and Guo et al. (2013) an improvement of the model by De Lucia \& Blaizot (2007). The Guo's models implement several new features: the separate evolution of sizes and orientations of gaseous and stellar discs, the size evolution of spheroids, tidal and ram-pressure stripping of satellite galaxies, and the disruption of galaxies to produce intra-cluster light. All three models include the effects of AGN feedback. The stellar masses of the semianalytical galaxies were estimated assuming a Chabrier (2003) initial mass function (IMF) for the three models. This is consistent with the IMF assumed in the observational works used to compare with the theoretical predictions.

\section{RESULTS}

We generate three galaxies catalogues using the Millennium database web (Lemson et al. 2006). Each catalogue corresponds to one different semi-analytical model as previously discussed. For each of the catalogues, we select all the massive galaxies at present-day as those objects with stellar masses between $8 \times 10^{10} M_{\odot}$ and $10^{13} M_{\odot}$.

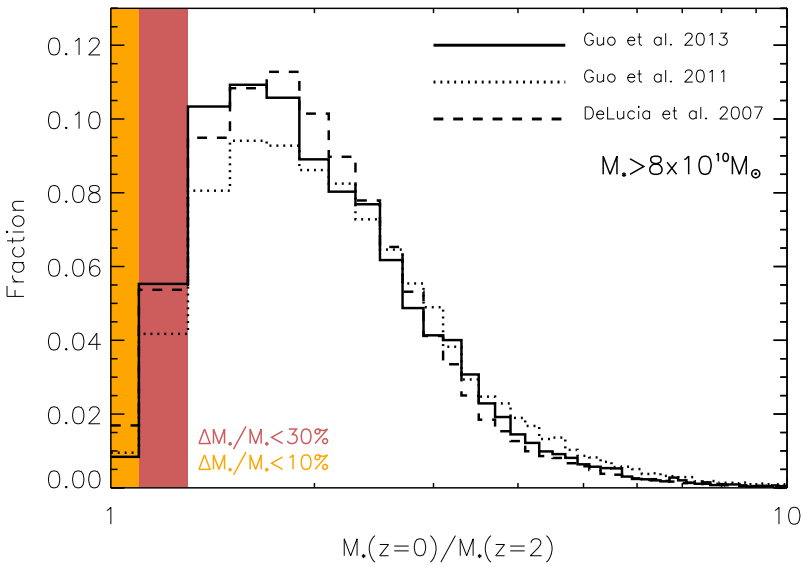

FIG. 1. - Fraction of present-day massive galaxies that already existed at $\mathrm{z} \sim 2$ as function of their relative increase of stellar mass. The different line styles stand for the three considered models. The color shaded areas show the fraction of galaxies that have increased their stellar masses less than a $10 \%$ and $30 \%$, respectively.

We define a relic as a galaxy in the present-day Universe that has barely increased its stellar mass since $z \sim 2$. In order to identify the possible candidates to relic galaxies, we use the merger tree structures to trace backwards in time the massive galaxies identified at $z \sim 0$, together with two conditions: i) the galaxy must be already form at $z \sim 2$, and ii) the galaxy mass at $z \sim 2$ has to be larger than the $90 \%$ or the $70 \%$, depending on the considered case, of the limit mass at the presentday $8 \times 10^{10} M_{\odot}$. Once all the candidate to relics are selected, it is possible to obtain their stellar mass increment at several redshift since $z \sim 2$. To have an estimation of the number of massive relics in the present-day Universe, we quantify the number of almost pure massive relics as the number of galaxies, among all the candidates, with an increase in their stellar mass less than $10 \%$ since $\mathrm{z}=2$. Additionally, we also explore the number of massive galaxies in the $\mathrm{z}=0$ Universe with an increase in stellar mass less than $30 \%$ since $z=2$. This last limit is selected to avoid that the galaxy has suffered a major (i.e. 1:3 or lower ratio) merger since $\mathrm{z}=2$ that could have altered their structure.

In Figure 1, we show the fraction of massive galaxies at $z \sim 0$ already present at $z \sim 2$ as a function of the relative increment of their stellar mass. All three considered models, show that roughly half of the massive galaxies already build at $z \sim 2$ have increased their masses at $z \sim 0$ more than a factor of two. Only a small fraction of the massive galaxies at the present epoch corresponds to massive galaxies at $z \sim 2$ that remain untouched or with a minor increase in their masses. In fact, less than a $2 \%(8 \%)$ of the massive galaxies already form at $\mathrm{z} \sim 2$ gained less than a $10 \%(30 \%)$ of their stellar mass since that epoch.

In Figure 2, we represent the redshift evolution of the ratio of the number of relic galaxies to the total number of massive galaxies (left panel) and the number density of relics for the three considered models (right panel). The total number of massive galaxies at each redshift account for all the massive galaxies that are progressively being incorporated to the family of mas- 

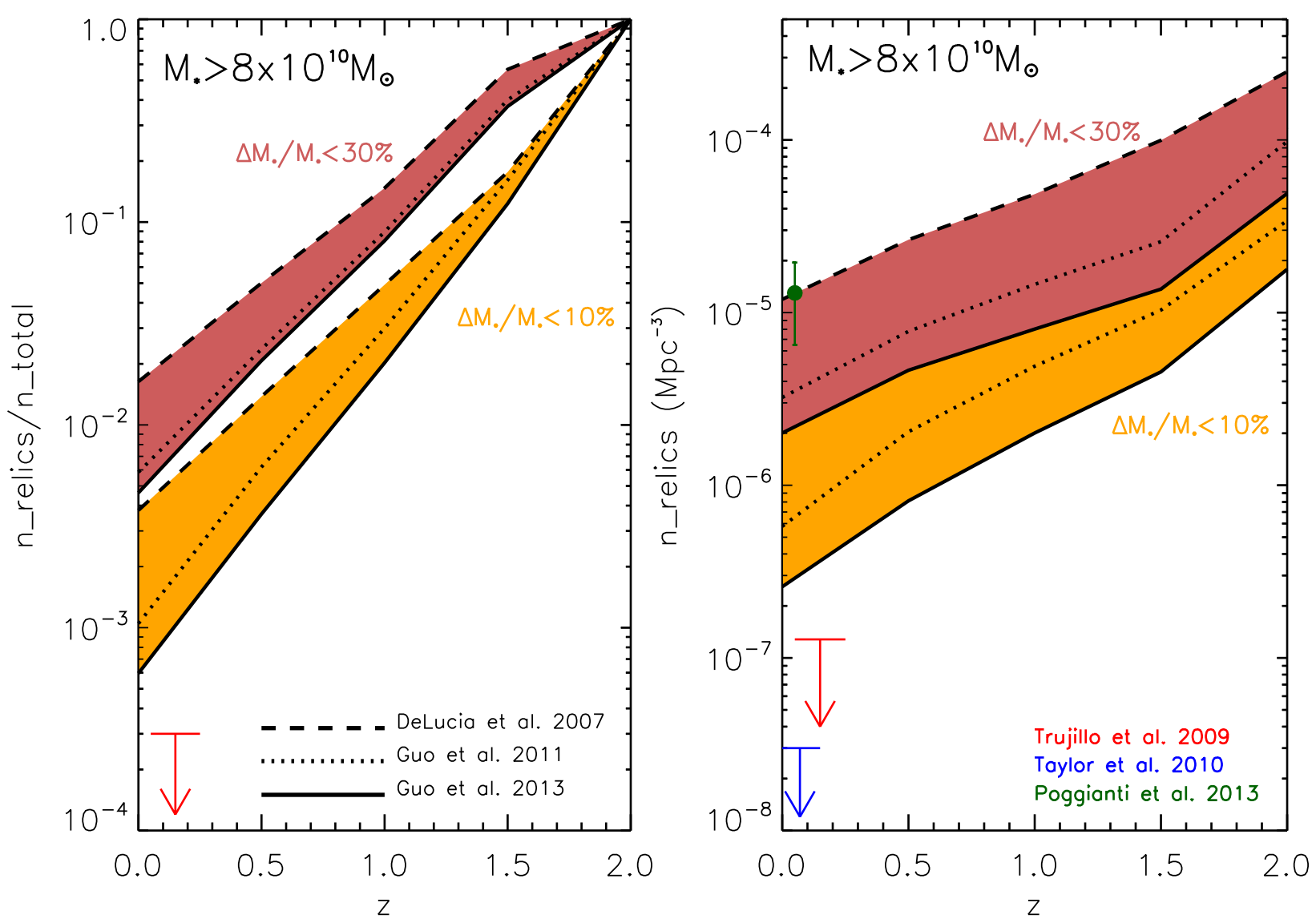

FIG. 2.- Left panel: redshift evolution of the ratio of the relic galaxies to the total number of massive galaxies. The three different lines represent the three considered models. Coloured areas, orange (orange-red) show galaxies that have increased their masses less than a 10\% (30\%) since $z \sim 2$. Right panel: redshift evolution of the comoving number density of relic galaxies. The lines and colour shaded areas stand as for the right panel. The red and blue arrow show the observational upper limits from Trujillo et al. (2009) and Taylor et al. (2010), respectively. The green point display the observational data from Poggianti et al. (2013).

TABLE 1

FraCtions AND NUMBER DENSITIES OF THE PRESENT-DAY MASSIVE GALAXY RELICS

\begin{tabular}{lllll}
\hline \hline $\begin{array}{l}\Delta M_{*} / M_{*} \\
(\text { since } \mathrm{z}=2)\end{array}$ & DeLucia et al. (2007) & Guo et al. (2011) & Guo et al. (2013) & Average \\
\hline & $\mathrm{n}_{\text {relics }} / \mathrm{n}_{\text {total }}$ at $z \sim 0$ & & & \\
\hline$<10 \%$ & 0.0038 & 0.001 & 0.0006 & 0.0018 \\
$<30 \%$ & 0.016 & 0.006 & 0.005 & 0.009 \\
\hline & Number density $\left(\mathrm{Mpc}^{-3}\right)$ & & & \\
\hline$<10 \%$ & $2.7 \times 10^{-6}$ & $5.8 \times 10^{-7}$ & $2.6 \times 10^{-7}$ & $1.2 \times 10^{-6}$ \\
$<30 \%$ & $1.2 \times 10^{-5}$ & $3.2 \times 10^{-6}$ & $2.0 \times 10^{-6}$ & $5.7 \times 10^{-6}$ \\
\hline
\end{tabular}

sive galaxies as cosmic time increases. No substantial differences appear between the three models. At all redshifts, De Lucia \& Blaizot (2007) model produces ratios of relic galaxies slightly higher than the other two models. As in Fig 1, we distinguish between two samples, those massive galaxies that have increased their relative stellar masses less than a 10\% and those others with a relative mass increment less than a $30 \%$. For the shake of completeness, we include the observational upper limits from Trujillo et al. (2009) and Taylor et al. (2010), and the observational data from Poggianti et al. (2013) [2.

The nowadays ratios of relic galaxies to total number of galaxies and the present-day number densities for the three different considered catalogues are summarized in Table 1.

\section{DISCUSSION}

As mention in the Introduction, to have an accurate estimation of the expected number density of massive

2 The number densities from Trujillo et al. (2009), Taylor et al. (2010) and Poggianti et al. (2013) are computed assuming a standar cosmology: $\Omega_{m}=0.3, \Omega_{\Lambda}=0.7$, and $H_{0}=70 \mathrm{kms}^{-1} M p c^{-1}$. 
relics in the present-day Universe is crucial to probe the minor merging channel of galaxy growth. Being the massive galaxies at high-z much more compact compared to current massive counterparts, a good proxy to identify massive galaxy relics in the nearby Universe is to search for massive compact galaxies with old stellar populations. The observed fraction of compact $\left(\mathrm{r}_{e}<1.5 \mathrm{kpc}\right)$ massive $\left(\mathrm{M}_{\star}>8 \times 10^{10} \mathrm{M}_{\odot}\right)$ galaxies with $\mathrm{z}<0.2$ in the present-day Universe (i.e. a good set of candidates to be relics of the early Universe) is $\sim 0.0003$ (Trujillo et al. 2009). At first glance this number is pretty similar to the fractions estimated in this Letter (particularly with those values obtained using the Guo et al. 2013 model). However, the number estimated by Trujillo et al. (2009) is uncertain for several reasons. The first one is that the ages of the compact galaxies found in that work are relatively young $(\sim 2$ Gyr $)$, this means that they can not be relics of the early Universe. In that sense, Trujillo et al. results should be understand as an upper limit of the true value (i.e. $<0.0003 ;<1.3 \times 10^{-7} M p c^{-3}$ ). Using a slightly different selection $\left(\mathrm{M}_{\star}>8 \times 10^{10} \mathrm{M}_{\odot}\right.$ and $\Delta \log \mathrm{r}_{e}<-0.4$ dex with repect to the SDSS stellar mass - size relation from Shen et al. 2003) and focusing only on red objects (i.e. more likely to be relics from the early Universe), Taylor et al. (2010) found only one dubious candidate at $0.066<\mathrm{z}<0.12$. This is 5000 times less than the expected number density of compact massive galaxies in the present-day Universe if the high-z massive objects were not evolve neither in size or mass (i.e. $\sim 1.5 \times 10^{-4} \mathrm{Mpc}^{-3}$ versus the number found in Taylor et al. $2010,<3 \times 10^{-8} \mathrm{Mpc}^{-3}$ )

Is it possible that both Trujillo et al. (2009) and Taylor et al. (2010) results can biased against the detection of massive relics in the present-day Universe? Taylor et al. (2010) have done an extensive study of the potential biases that affect the SDSS spectroscopic sample and found that their results can not be explained as consequence of incompleteness. In a recent work, Poggianti et al. (2013) applying the same selection that in Trujillo et al. (2009) found 4 old compact massive galaxies in 30.88 $\mathrm{deg}^{2}$ within $0.03<\mathrm{z}<0.11$ using the PM2GC (Calvi et al. 2011) sample. This is equivalent to a number density of $1.3 \times 10^{-5} \mathrm{Mpc}^{-3}$. This number is significantly larger than the numbers quoted in Trujillo et al. (2009) and Taylor et al. (2010) studies. Poggianti et al. (2013) results are based in a sample with a larger spectroscopic completeness. However, the different spectroscopic completeness of the SDSS survey compared to the PM2GC can not explain a factor of at least 100 in the discrepancy among these works. Poggianti et al. (2013) indicates that only $25 \%$ of their compact galaxies are not identified spectroscopically in the SDSS. It is clear that a more detailed analysis is necessary to solve this enormous discrepancy.

Although the expected fraction of massive relics galaxies change by a factor of 5 among the different semianalytical models, the change in the number density can be as high as a factor of 10. For the discussion and the comparison with the observations, we take the average values of the different models for the fraction and the number density of massive relics. The results based on the SDSS survey (Trujillo et al. 2009; Taylor et al. 2010) found a significant less (more than a factor of 10) number of relics than what is predicted by the models. If these observations are confirmed in the future this will be hardly understood within the merging scenario. Due to its stochastic nature, there should be a number (small but measurable) of relics in the nearby Universe that remains undetected. Again, if confirmed, the absence of compact massive relic galaxies in the nearby Universe will be a strong indication that the growth mechanisms of the massive galaxies need something else than merging to produce the observed massive population or that the number of mergers is much larger than what it is theoretically predicted. This is odd, as we know that the number of satellites among massive galaxies at all redshift is overpredicted by those simulations (see e.g. Quilis \& Trujillo 2012). On the contrary, if the number density of massive relics galaxies is closer to the values quoted by Poggianti et al. (2013) then the number of merging seems to be lower than what is theoretically expected. Once more, it is urgent to understand the discrepancy between the observational results to put a stringent constraint to the question.

The authors thank Bianca Poggianti for clarifying some aspects of her work and the anonymous referee for useful comments and criticism. This work was supported by the Spanish Ministerio de Economía y Competitividad (MINECO)(grants AYA2010-21322-C03-01 and AYA2010-21322-C03-02) and the Generalitat Valenciana (grant PROMETEO-2009-103). The Millennium Simulation databases used in this paper and the web application providing online access to them were constructed as part of the activities of the German Astrophysical Virtual Observatory.

\section{REFERENCES}

Bezanson, R., van Dokkum, P. G., Tal, T., Marchesini, D., Kriek, M., Franx, M. \& Coppi, P., 2009,ApJ, 697, 1290

Buitrago, F., Trujillo, I., Conselice, C. J., et al. 2008, ApJ, 687, L61

Calvi, R., Poggianti, B. M., \& Vulcani, B. 2011, MNRAS, 416, 727 Chabrier G., 2003, PASP, 115, 763

Daddi, E. et al., 2005, ApJ, 626, 680

Davis M., Efstathiou G., Frenk C. S., White S. D. M., 1985, ApJ, 292,371

De Lucia G., Blaizot J., 2007, MNRAS, 375, 2

Feldmann, R., Carollo, C. M., Mayer, L., Renzini, A., Lake, G., Quinn, T., Stinson, G. S., Yepes, G., 2010, ApJ, 709, 218

Ferré-Mateu, A., Vazdekis, A., Trujillo, I., et al. 2012, MNRAS, 423,632
Guo Q., White S., Boylan-Kolchin M., De Lucia G., Kauffmann G., Lemson G., Li C., Springel V., Weinmann S., 2011,MNRAS, 413, 101

Guo, Q., White, S., Angulo, R. E., et al. 2013, MNRAS, 428, 1351

Hopkins, P. F., Bundy, K., Murray, N., Quataert, E., Lauer, T. R., Ma, C.-P., 2009, 398, 898

Komatsu, E., Smith, K. M., Dunkley, J., et al. 2011, ApJS, 192, 18

Lemson G. and the Virgo Consortium, 2006, astro-ph/0608019

Naab, T., Johansson, P. H.. Ostriker, J. P., 2009, ApJ, 699, L178

Oser, L., Naab, T., Ostriker, J. P., Johansson, P. H., 2012, ApJ, 744,630

Poggianti, B. M., Calvi, R., Bindoni, D., et al. 2013, ApJ, 762, 77 Quilis, V., \& Trujillo, I. 2012, ApJ, 752, L19 
Shen, S., Mo, H. J., White, S. D. M., et al. 2003, MNRAS, 343, 978

Sommer-Larsen, J. \& Toft, S., 2010, ApJ, 721, 1755

Springel V., White S. D. M., Tormen G., Kauffmann G., 2001, MNRAS, 328, 726

Springel V. et al., 2005, Nat, 435, 629

Taylor, E. N., Franx, M., Glazebrook, K., et al. 2010, ApJ, 720, 723
Trujillo I., et al., 2006, MNRAS, 373, L36

Trujillo, I., Conselice, C. J., Bundy, K., et al. 2007, MNRAS, 382, 109

Trujillo, I., Cenarro, A. J., de Lorenzo-Cáceres, A., et al. 2009, ApJ, 692, L118 